\title{
Transmissão de dados agrometeorológicos através de um módulo para rastreamento de veículos automotores
}

\author{
Fábio Iaione, Diogo Fernando Trevisan e Cleiton Schwinn
}

\begin{abstract}
Resumo-As plantações são afetada por fungos, cujo desenvolvimento depende do clima. Para diminuir a aplicação de fungicidas, existem modelos matemáticos de previsão que fornecem um índice de infecção, calculado utilizando-se parâmetros climáticos. Nos sistemas de previsão, estações meteorológicas enviam dados via rede de telefonia celular, para um computador que executa o modelo. Onde não há cobertura da rede celular, o sistema fica prejudicado. O objetivo desse trabalho foi usar um módulo para rastreamento de veículos na transmissão de dados agrometeorológicos via satélite. Nos testes, todas as mensagens contendo dados climáticos foram recebidas sem erro.
\end{abstract}

Palavras-chave-Transmissão via satélite, Dados agrometeorológicos, Sistemas de previsão de doenças em plantas.

Abstract-Plantations are affected by fungi, whose development depends on the weather. To reduce the application of fungicides, there are forecast mathematical models that provide an index of infection calculated using climatic parameters. In forecast systems, meteorological stations send data via the mobile phone network to a computer that runs the model. Where there is not coverage of the mobile phone network, the system is impaired. The aim of this work was to use a module for tracking vehicles in agrometeorological data transmission via satellite. In tests, all messages containing climate data were received without error.

Keywords-Satellite transmission, agrometeorological data, prediction systems for diseases in plants.

\section{I.INTRODUÇ̃̃O}

A produção agrícola é constantemente afetada por fungos que se desenvolvem em função das condições climáticas (temperatura, umidade e outras). Sem o conhecimento das condições climáticas, a aplicação de fungicidas deve ser efetuada periodicamente, resultando em prejuízos financeiros e ecológicos. Para que isto não ocorra, existem vários modelos matemáticos que calculam um índice de infecção para as doenças e auxiliam os agricultores na determinação do momento de pulverizar a plantação, diminuindo a quantidade de aplicações químicas [1] - [5]. Normalmente, os sistemas de previsão de doenças em plantas são formados por pequenas estações meteorológicas [6], [7] espalhadas na plantação que

Fábio Iaione, Diogo Fernando Trevisan e Cleiton Schwinn, Faculdade de Ciências Exatas e Tecnologia, Universidade Federal da Grande Dourados, Dourados, MS, Brasil, E-mails: fabio.iaione@ufgd.edu.br, pancatrevisan@gmail.com, schwinn.mju@gmail.com enviam dados climáticos através da rede de telefonia celular a um computador central, o qual executa o modelo matemático e determina o índice de infecção que é usado na decisão de aplicar ou não os fungicidas.

Onde não há cobertura da rede celular, a coleta de dados deve ser feita manualmente e, mais tarde, os dados devem ser enviados através da internet ao computador central, implicando em aumento de tempo e custos para a coleta das informações. Uma possível solução para este problema é transmitir os dados das estações ao computador central usando um módulo de transmissão de dados via satélite (MTDS). O satélite, do ponto de vista de transmissão, é uma simples estação repetidora dos sinais recebidos da terra que são detectados, deslocados em freqüência, amplificados e retransmitidos de volta à terra [8], [9].

A maioria dos satélites de comunicação é geoestacionário e orbita ao redor da terra movendo-se na mesma direção e velocidade desta, permanecendo imóvel em relação a um ponto na superfície terrestre. Esses satélites estão posicionados acima da linha do equador, a $35.786 \mathrm{~km}$ de altitude, e movendo-se a $28.000 \mathrm{Km} / \mathrm{h}$ [8], [9].

O sistema Inmarsat é o mais usado e um dos pioneiros em comunicação via satélite. Este sistema conta com dezenas de satélites de diferentes gerações, cobrindo praticamente todo o globo terrestre, e oferecendo diversos serviços [10].

Diante dessas informações, o objetivo do trabalho foi selecionar um MTDS, utilizado para rastreamento de veículos automotores, estudar os protocolos utilizados pelo mesmo e desenvolver um sistema para transmitir dados climáticos da lavoura (Figura 1).

\section{II.Metodologia}

O primeiro passo foi realizar um levantamento dos MTDS existentes no mercado. Foram encontrados 34 (trinta e quatro) modelos de MTDS e, após uma pré-seleção, selecionou-se 9 (nove) MTDS. Através de uma análise desses 9 (nove) MTDS, levando-se em consideração as dimensões, peso, tensão de alimentação, consumo de energia, tipo de interface e classe de uso, selecionou-se 4 (quatro) MTDS. Dentre os selecionados estavam o modelo TT-3026L/M easytrack (Thrane \& Thrane); o GSP-2900 (Globalstar); o EXPLORER 110 (Thrane \& Thrane) e por fim o SAT-201 (Satamatics), que eram mais adequados para a aplicação em questão. Após uma análise do custo e dimensões desses 4 (quatro) MTDS, 
selecionou-se o Satamatics SAT-201 como o mais apropriado (custo aproximado de US\$ 800).

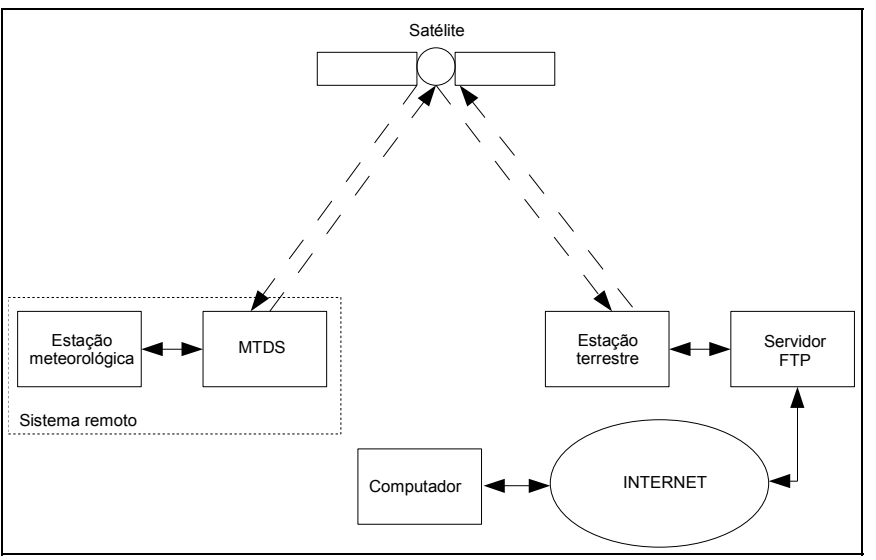

Fig. 1. Estação meteorológica conectada ao MTDS, que envia os dados para o satélite. Os dados são retransmitidos do satélite de volta para a terra e são disponibilzados por um servidor FTP que pode ser acessado através da internet.

O MTDS selecionado possui dimensões relativamente reduzidas, tendo $112 \mathrm{~mm}$ de diâmetro, $46 \mathrm{~mm}$ de altura e pesando apenas $350 \mathrm{~g}$ (Figura 2).

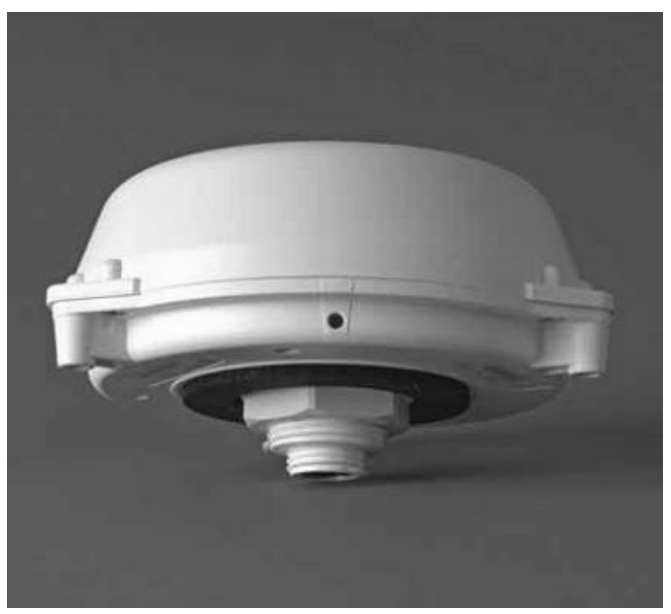

Fig. 2. Módulo de transmissão de dados via satélite modelo SAT-201 (retirado de [11]).

O consumo de energia é de $5 \mathrm{~W}$ quando está enviando uma mensagem, 1,5 W quando está recebendo e $0,5 \mathrm{~mW}$ quando está no modo de espera. A antena de comunicação está embutida dentro do próprio MTDS que deve ser instalado a céu aberto. Em sua parte inferior existe um conector de 12 (doze) pinos para comunicação e fornecimento de energia. Ele comunica-se com o satélite utilizando a banda L (1,6 $\mathrm{GHz}$ ) e o serviço Inmarsat $\mathrm{D}+$. A Tabela I mostra as principais características desse módulo [11].

As mensagens enviadas pelo MTDS são disponibilizadas em um servidor FTP (na pasta saídas) na forma de arquivos texto (.txt). Cada mensagem enviada contém, além dos dados transmitidos, a data e hora em que saiu do MTDS, permitindo assim uma ordenação das mensagens na recepção. Da mesma forma, as mensagens enviadas para o MTDS devem ser colocadas no servidor FTP (pasta entradas) como arquivo de texto. Nesses arquivos de texto, cada dado (byte) é representado em hexadecimal através de dois caracteres.

TABELA I

PRINCIPAIS CARACTERÍSTICAS DO MÓDULO DE TRANSMISSÃO DE DADOS VIA SATÉLITE SELECIONADO.

\begin{tabular}{|c|c|}
\hline Taxa de transmissão & $\approx 10 \mathrm{bps}$ \\
\hline $\begin{array}{l}\text { Mensagem de } \\
\text { transmissão }\end{array}$ & Até 10,5 bytes ( 84 bits) \\
\hline Taxa de recepção & $\approx 9 \mathrm{bps}$ \\
\hline Mensagem de recepção & Até 1000 bits \\
\hline Interface de comunicação & $\begin{array}{l}\text { Serial (RS232 assíncrona), } 9600 \mathrm{bps} \text {, sem } \\
\text { paridade, } 8 \text { bits de dados e } 1 \text { bit de parada. }\end{array}$ \\
\hline Tensão de alimentação & $9,6 \mathrm{~V}$ a $32 \mathrm{~V}$ (corrente contínua) \\
\hline Ambiente de utilização & $\begin{array}{l}\mathrm{T}=-40^{\circ} \mathrm{C} \mathrm{a}+70^{\circ} \mathrm{C}, \mathrm{UR} \leq 95 \% @+40^{\circ} \mathrm{C}, \\
\mathrm{IP} 66\end{array}$ \\
\hline
\end{tabular}

No primeiro teste realizado, o MTDS encontrava-se ligado através da porta serial RS232 a um notebook e a céu aberto, e os comandos foram enviados ao MTDS através do aplicativo Hyperterminal do Windows XP.

Após este teste, a estrutura das mensagens e os protocolos de comunicação foram conhecidos, e o desenvolvimento do aplicativo para acessar o servidor FTP teve início. Este programa foi totalmente escrito na linguagem JAVA [12] - [15], utilizando-se o Sun JDK (Java Development Kit) versão 6, com a IDE JCreator LE 3.5, no sistema operacional Microsoft Windows XP. A comunicação com o servidor é feita através dos métodos implementados nas bibliotecas Apache Commons Net e Apache Oro [16], as quais implementam as classes java.net, e facilitam a comunicação entre computadores, ou mesmo conexões com a internet. A função do programa é basicamente conectar-se ao servidor, fazer download dos arquivos e processá-los, gerando uma tabela com os dados obtidos. Esta tabela é salva em disco, podendo futuramente ser visualizada.

Para comunicação com o MTDS via cabo, utilizou-se um Módulo de Computação Embarcada (MCE) que foi programado para comportar-se como uma estação meteorológica. Este MCE é dotado de vários componentes eletrônicos indispensáveis para a conexão com o MTDS, além de um sensor de temperatura. Entre os principais componentes do MCE estão um microcontrolador da família MCS-51 [17] - [20], modelo 80C552, e uma memória RAM (32 KB) que armazena o programa (firmware) a ser executado. Um visor de cristal líquido (2 linhas x 16 caracteres) permite a visualização de texto durante a execução do firmware. O firmware foi desenvolvido em linguagem $\mathrm{C}$ [21] - [23], com a IDE Proview32, sobre o sistema operacional Microsoft Windows XP. Basicamente, o firmware implementa um relógio, pois este fica aguardando a ocorrência de uma interrupção que ocorre a cada décimo de segundo, ou seja, a cada dez interrupções se tem um segundo, e assim o relógio pode funcionar corretamente. A cada $2 \mathrm{~min}$ é realizada uma leitura de temperatura e uma mensagem, contendo essa informação e outros dados climáticos fictícios, é enviada pela porta serial ao MTDS.

O teste de todo sistema ocorreu em duas etapas. A primeira etapa iniciou às $14 \mathrm{~h} 53 \mathrm{~min} 0 \mathrm{~s}$ e terminou uma hora depois. Nesse teste, o aplicativo para acessar o servidor FTP não pode ser testado em tempo real, devido a uma 
indisponibilidade temporária de conexão do computador (Figura 1) com a internet, a qual é parte essencial do sistema. O MTDS foi colocado a $6 \mathrm{~m}$ de distância do telhado do prédio, conectado ao MCE que simulava a estação meteorológica, o qual disparava mensagens de dados a cada dois minutos para o MTDS. Tais dados continham a hora e minuto do relógio implementado no $\mathrm{MCE}$, que iniciava em $0 \mathrm{~h}$ 0min 0s, a temperatura do ar medida, os valores de mais 8 (oito) parâmetros climáticos simulados, pois o MCE utilizado possuía apena 1 (um) sensor de temperatura, e um byte de LRC (Longitudinal Redundancy Check).

A segunda etapa do teste, necessária devido ao problema ocorrido com a internet na primeira etapa, teve inicio às $16 \mathrm{~h}$ $44 \mathrm{~min} 30 \mathrm{~s}$ e também durou aproximadamente 1 hora, sendo que o programa foi testado em tempo real. Em cada etapa de teste foram enviadas aproximadamente 30 mensagens.

Em todos os testes realizados, a energia elétrica para funcionamento do MTDS foi obtida através de uma fonte de tensão (corrente contínua) não regulada de $12 \mathrm{~V}$ (wall cube).

\section{III.RESULTADOS}

No total, sessenta e três mensagens foram enviadas no teste, e todas chegaram sem erro de LRC. O aplicativo desenvolvido processou todas elas, separando corretamente cada dado. Como o fuso horário do estado do Mato Grosso do Sul é de $-4 \mathrm{~h}$ em relação à Greenwich, a data contida nos arquivos foi corrigida.

A Figura 3 mostra a distribuição de freqüência do tempo para as mensagens serem enviadas, obtida na segunda etapa do teste.

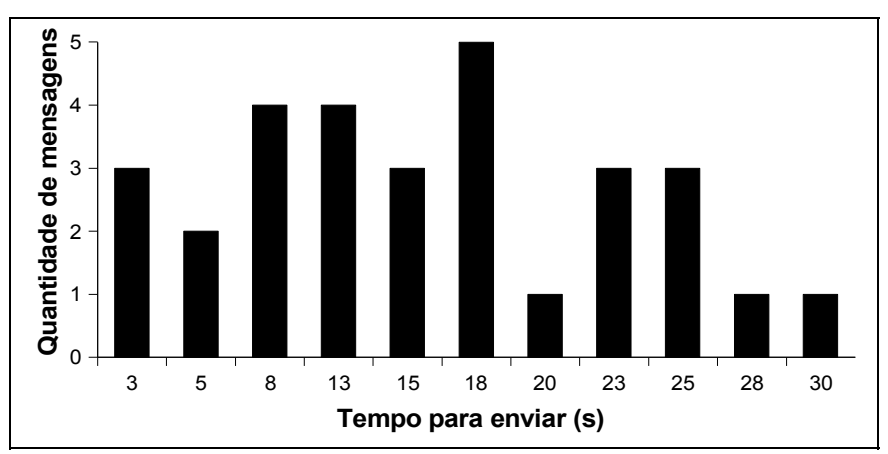

Fig. 3. Distribuição de frequência do tempo que a mensagem leva saindo do MCE até ser enviada pelo MTDS (segunda etapa de teste).

Na primeira etapa de teste do sistema, o tempo mínimo de envio das mensagens foi de $15 \mathrm{~s}$, o tempo máximo foi de $8 \mathrm{~min}$ e $25 \mathrm{~s}$, tendo como tempo médio 2 min e $28 \mathrm{~s}$ e desvio padrão de 2 min e 32 s. Já para as mensagens da segunda parte do teste, o tempo mínimo de envio foi $3 \mathrm{~s}$, o tempo máximo $30 \mathrm{~s}$, a média $15 \mathrm{~s}$ e o desvio padrão $7 \mathrm{~s}$.

\section{IV.Discussão E Conclusão}

O MCE funcionou corretamente simulando a estação meteorológica e o aplicativo desenvolvido para acessar o servidor FTP também, fazendo download das mensagens, apagando os arquivos no servidor e processando os dados recebidos. Portanto, implementou-se um protótipo de sistema para transmissão de dados climáticos da lavoura via satélite.

Em relação a diferença entre os tempos médios para envio das mensagens, obtidos na primeira e segunda etapas de teste, acredita-se que ocorreu em função da variação do tráfego no sistema de comunicação via satélite, e que devido a presença de um buffer interno no MTDS para 9 (nove) mensagens, os tempos maiores não causaram problemas.

Em relação a disponibilidade do sistema, a operadora informou que não há indisponibilidade prevista para o estado do Mato Grosso do Sul e outras regiões do Brasil. Além disso, mesmo que ocorra uma indisponibilidade, os dados climáticos ficam armazenados em uma memória interna que toda estação meteorológica possui. O número de mensagens transmitidas, apesar de pequeno, permitiu uma análise inicial da confiabilidade do sistema. Como não existe indisponibilidade prevista, acredita-se que os resultados se confirmem para centenas ou até milhares de mensagens. Cabe observar que a indisponibilidade citada na primeira etapa de teste do sistema completo refere-se à "queda" da conexão entre o computador, executando o aplicativo desenvolvido, e a internet, e não do servidor FTP (Figura 1).

$\mathrm{O}$ fato de não ter ocorrido nenhuma alteração nos dados transmitidos (dados corrompidos) pode ser atribuído a existência de detecção de erros no próprio sistema MTDS - satélite - servidor FTP, que é "invisível" para o usuário. Aliado a isso, tem-se mensagens com pequena quantidade de dados (252 bytes), o que diminui a probabilidade de erros, e ainda, uma comunicação entre $\mathrm{MCE}$ e MTDS via cabo de pequena dimensão (6 m) e com uma taxa de transmissão relativamente baixa (9600 bps).

Em uma aplicação real, com o MTDS localizado junto a uma estação meteorológica na lavoura, a fonte de energia elétrica para o MTDS pode ser obtida da própria estação, quando possível, ou através de uma bateria de $12 \mathrm{~V}$ recarregável, conectada a um painel solar fotovoltaico, devidamente dimensionados.

O MTDS utilizado é à prova d'água e possui uma superfície lisa e convexa na parte superior (plástico), portanto, acreditase que ocorra pouca deposição de sujeira e que a água da chuva realize a limpeza dessa superfície.

Considerando-se os resultados dos testes, pode-se concluir que pequenos MTDS, usados principalmente em aplicações de rastreamento de veículos automotores, podem ser utilizados para transmissão de parâmetros climáticos da lavoura, apesar de suas limitações, como taxa de transmissão reduzida (11 bps para o MTDS utilizado).

\section{Agradecimentos}

Os autores agradecem a Universidade Federal da Grande Dourados, pelo apoio financeiro, e a empresa Innovatis Tecnologia pelo empréstimo de um MTDS, sem o qual esse trabalho não poderia ter sido realizado. 


\section{REFERÊNCIAS}

[1] J. R. Wallin, "Summary of recent progress in predicting late blight epidemics in United States and Canada", American Potato Journal, v. 39, pp. 306-312, 1962.

[2] R.A. Krause e L. B. Massil, "Predictive Systems: modern aproaches to disease control. Ann. Rev.”, Phytopat, v. 13, pp. 31-47, 1975.

[3] J. K. Kochman, "The effect of temperature on development of soybeans rust (Phakopsora pachyrhizi)", Australian Journal of Agricultural Research, v.30, n.2, pp. 273-277, 1979.

[4] J. S. Melching, W. M. Dowler, D. L. Koogle, M. H. Royer, "Effects of duration, frequency, and temperature of leaf wetness periods on soybean rust", Plant Disease, v. 73, n. 2, pp. 117- 122, 1989.

[5] E. M. Del Ponte, C. V. Godoy, M. G. Canteri, E. M. Reis, X. B. Yang, "Models and applications for risk assessment and prediction of asian soybean rust epidemics", Fitopatologia Brasileira, 31(6), pp. 533-544, nov-dez 2006.

[6] F. Iaione, D. G. Lima, F. R. Gassem, "Equipamento para coleta de dados e previsão de doenças na lavoura", Prêmio Jovem Cientista 1995: Publicação Resumida dos Trabalhos Vencedores, 1999.

[7] F. Iaione, Coletor Eletrônico de Parâmetros Ambientais. BR m. PI9606372-6, 9 dez. 1996, 20 maio 2008.

[8] B. R. Elbert, The Satellite Communication Applications Handbook. Artech House, 2004.

[9] Teleco, Informação em telecomunicações. Disponível em: http://www.teleco.com.br, Maio 2008.

[10] Inmarsat, The Mobile Satellite Company, Disponível em: http://www.inmarsat.com, Junho 2008.

[11] Satamatics, SAT-201 User Manual. Satamatics, 2007.

[12] C. C. Mark, W. S. Griffit, F. A. IASI, 1001 Java Programmer's Tips. Editora Jamsa Press, 1997.

[13] H. M. Deitel e P. J. Deitel, Java - Como Programar. Editora PrenticeHall, 2005.

[14] C. Horstmann, Big Java. Editora Bookman, 2004.

[15] Sun Microsystems, JavaTM 2 Platform, Standard Edition v 1.4.2 API Specification, Disponível em: http://java.sun.com/j2se/1.4.2/docs/api, Julho 2003.

[16] The Apache Software Foundation, Commons Net 1.5.0 - SNAPSHOT Disponível http://commons.apache.org/net/apidocs/index.html, Julho 2008.

[17] I. S. Mackenzie, The 8051 microcontroler. Macmillan Pub Co, 1994.

[18] D. E. C. Nicolosi e R. B. Bronzeri, Microcontrolador $8051 \mathrm{com}$ linguagem C: Prático e Didático - Família AT89S8252 Atmel. Editora Érica, 2005 .

[19] V. P. Silva Jr, Aplicações Práticas do Microcontrolador 8051. Editora Érica, 2006.

[20] D. E. C. Nicolosi, Laboratório de Microcontroladores Família 8051 Treino de Instruções, Hardware e Software. Editora Érica, 2002.

[21] B.W. Kernighan e D. M. Ritchie, The C Programming Language. Prentice Hall, 1998.

[22] B. S. Gottfried, Programando em C. Markron Books, 1990.

[23] H. Schildt, $C$ completo e total. Pearson, 2006. 